UdeM-LPN-TH-94-188

hep-th/9405027

\title{
Parity violation, anyon scattering and the mean field approximation
}

\author{
Didier Caenepeel and Richard Mackenzie \\ Laboratoire de Physique Nucléaire \\ Université de Montréal \\ Montréal, Qué, H3C 3J7 \\ Canada
}

\begin{abstract}
Some general features of the scattering of boson-based anyons with an added non-statistical interaction are discussed. Periodicity requirements of the phase shifts are derived, and used to illustrate the danger inherent in separating these phase shifts into the well-known pure Aharanov-Bohm phase shifts, and an additional set which arise due to the interaction. It is proven that the added phase shifts, although due to the non-statistical interaction, necessarily change as the statistical parameter is varied, keeping the interaction fixed. A hard-disk interaction provides a concrete illustration of these general ideas.
\end{abstract}


In the latter part of the paper, scattering with an additional hard-disk interaction is studied in detail, with an eye towards providing a criterion for the validity of the mean-field approximation for anyons, which is the first step in virtually any treatment of this system. We find, consistent with previous work, that the approximation is justified if the statistical interaction is weak, and that it must be more weak for boson-based than for fermion-based anyons. 
It is well known that in two space dimensions the possibility of fractional statistics exists [1]. The simplest way of describing these fractional-statistics particles, anyons, is to start with conventional particles and attach fictitious "statistical" point charges and fluxes to the particles $[2,3]^{\dagger}$. The resulting Aharonov-Bohm (AB) interaction mimics a change in statistics; for example, adopting a pathintegral point of view, a path involving a winding of one particle around another acquires an additional contribution to its action from the statistical interaction proportional to the change in relative angle, independent of the details of the path.

Depending on whether one starts from bosons or fermions, the strength of the statistical interaction measures the departure of the statistics from the "base" statistics. Evidently, one has a periodicity requirement: starting from bosons, for example, one eventually reaches a statistical interaction which transmutes them into fermions; one requires that the dynamics of bosons transmuted to fermions agree with that of the fermions themselves, all else being equal.

Anyons have been studied in great detail, mostly due to applications in the fractional quantum Hall effect and to the prospect of a new mechanism of superconductivity [4]. Since the statistical interaction is rather difficult to handle, the starting point is usually a "mean-field approximation" (MFA), which consists in replacing the flux carried by each anyon by a uniform magnetic field with the same average value [5]. This reduces the many-anyon problem to a system of many conventional particles in a uniform magnetic field plus an interaction which is a sum of the conventional (e.g., Coulomb) interaction between anyons and an interaction

\footnotetext{
$\dagger$ In this article, we consider only electrically neutral particles; thus any reference to charge, flux, etc., is understood to refer to the statistical quantities.
} 
term due to the MFA (equal to the point magnetic fields of the individual anyons minus the mean field).

At an intuitive level, since a sum of $\delta$-functions is exceedingly non-uniform, the validity of the MFA merits some scrutiny. Several arguments have been proposed, suggesting that the approximation should be valid if the statistical interaction is weak. One can, for instance, devise a criterion for validity of the MFA via a selfconsistency argument [6,9]. After making the MFA, particles move in circular orbits due to the uniform magnetic field. One can then evaluate the number of particles $Q$ contained inside a typical orbit and express it in terms of the strength of the statistical interaction $\alpha$ (normalized so that $\alpha=1$ transmutes bosons to fermions and vice versa). When $Q$ is sufficiently large, the granularity of the statistical magnetic field is unimportant and the MFA is deemed valid. One finds $[6,7,8,9]$ $Q \sim \alpha^{-2}$ for fermion-based anyons and $Q \sim \alpha^{-1}$ for boson-based anyons, yielding the following criterion of validity:

$$
\begin{array}{cl}
\alpha \ll 1 & \text { boson-based anyons } \\
\alpha^{2} \ll 1 & \text { fermion-based anyons }
\end{array}
$$

Thus, we see that the approximation is valid near Bose statistics for boson-based anyons, and near Fermi statistics for fermion-based anyons; the difference in powers of $\alpha$ indicates that one can be slightly further from conventional statistics for fermion-based anyons before the approximation breaks down.

In a recent paper [10], another means of justifying the MFA which does not rely on self-consistency was explored. The argument, alluded to in Ref. [11], consists in using parity violation in the scattering of anyons [12]. This results in an asymmetry in the scattering cross-section of two anyons, which allows one to evaluate a 
mean scattering angle for a typical anyon trajectory. From this, one can calculate the mean radius of the resulting quasi-circular orbit and extract (as in the selfconsistent argument described above) the number of particles $Q$ contained inside a typical orbit. The procedure, carried out for a system of boson-based anyons, yields the same result as found in the self-consistent argument described above, i.e., $Q \sim \alpha^{-1}$, recovering the criterion for validity of the MFA, $\alpha \ll 1$.

One peculiarity of the above "asymmetric-scattering" approach is that it is necessary to introduce a conventional interaction between the anyons. In [10], this interaction was parameterized by introducing a phase shift put into the lowest partial wave. The MFA result was recovered for "generic" phase shift, i.e., for one not near a multiple of $\pi$.

At a purely pragmatic level, this need for a conventional interaction arises because the scattering of two free anyons does not violate parity [12], and thus the mean radius of curvature calculated as above would be infinite. At a more profound level, it was conjectured [10] that for free anyons, the motion of a single anyon in an anyon gas is not correctly analyzed by regarding it as a sequence of individual scatterings (thus ignoring interference effects between subsequent scatterings). This conjecture is supported by a study of scattering of a charged particle off a semi-infinite rectangular lattice of flux tubes [13], wherein it was found that in the limit where the flux per anyon goes to zero, the scattering agrees with the motion of a particle in a uniform magnetic field (and hence with that of the MFA).

If this conjecture is correct, it is interesting that the asymmetric-scattering approach of [10] (which did not take into account multiple scatterings) seemed to work so well. Apparently, the coherence found in free anyon scattering which 
necessitates the inclusion of interference between subsequent scatterings is absent in the scattering of anyons with an additional interaction.

Ideally, as suggested in [13], it would be interesting to study the scattering of one anyon off a random array of flux tubes (more closely approximating the motion of an anyon in a gas of anyons) to see if the result of [13] persists.

Since such studies of multiple scatterings appear exceedingly difficult, it is worthwhile exploring further the validity of the MFA in a situation where the statistical interaction is supplemented by a conventional interaction, ignoring multiple scatterings. In this paper, we apply the asymmetric-scattering method to a more realistic system, namely, a gas of anyons with hard-disk repulsion. (Indeed, as we will show, parameterizing an added conventional interaction by a phase shift in the lowest partial wave is dangerously misleading in a rather subtle way, since it implies that the conventional interaction itself violates parity.) The scattering

of two such anyons exhibits clearly an asymmetry in the cross-section [14] due to parity violation, allowing us to calculate a mean radius of curvature for a typical orbit, and thus to deduce a criterion for the validity of the MFA. In the course of the analysis, we will present some peculiarities in the partial wave decomposition for anyons, which sheds some light on a seemingly bizarre situation arrived at in $[12]$

\section{Generalities}

To begin, it is perhaps worthwhile to discuss briefly the familiar case of conventional (non-AB) scattering, in order to set up notation, etc. The relative Hamil- 
tonian for two particles interacting via a potential $V(\mathbf{r})$ is

$$
H=-\frac{1}{2}\left(\frac{\partial^{2}}{\partial r^{2}}+\frac{1}{r} \frac{\partial}{\partial r}+\frac{1}{r^{2}} \frac{\partial}{\partial \theta^{2}}\right)+V(\mathbf{r}) .
$$

A scattering solution is sought of the form of an incident wave plus a scattered wave:

$$
\psi(r, \theta)=e^{i k r \cos \theta}+\frac{e^{i k r}}{\sqrt{r}} f(\theta)
$$

the scattering amplitude $f(\theta)$ being related to the scattering cross-section in the usual way, $d \sigma / d \theta=|f(\theta)|^{2}$.

If the particles are identical bosons, the scattering amplitude is in fact $F(\theta)=$ $f(\theta)+f(\theta-\pi)$, whereas for fermions it is $\tilde{F}(\theta)=f(\theta)-f(\theta+\pi)$.

With parity $\mathbf{P}$ defined as $(r, \theta) \rightarrow(r,-\theta)$, it is clear that if $V$ is an even function of $\theta$, the Hamiltonian respects $\mathbf{P}$, and both $f(\theta)$ and the cross-section will be even functions of $\theta$. If the potential is cylindrically symmetric, and a partial wave expansion can be performed:

$$
f(\theta)=\frac{1}{\sqrt{2 \pi i k}} \sum_{m=-\infty}^{\infty} e^{i m \theta}\left(e^{2 i \delta_{m}}-1\right) .
$$

Since cylindrical symmetry implies $\mathbf{P}$ invariance, $\delta_{-m}=\delta_{m}$. This implies, among other things, that $F$ and $\tilde{F}$ are sums over even and odd values, respectively, of $m$, and also the familiar result that fermions cannot scatter at angle $\pi / 2$ (equally valid, under normal circumstances, in two or three dimensions.)

Indeed, these 'normal circumstances' are so ubiquitous that any suggestion that identical fermions could scatter at angle $\pi / 2[12]$ seems at first downright 
blasphemous. It is perhaps worth mentioning, therefore, that with an unusual interaction fermions can easily be made to scatter at angle $\pi / 2$ : a fairly silly but perfectly valid example would be 'hard-rectangle' scattering in the classical limit, where the infinite rectangular potential barrier is skewed at an angle $\pi / 4$ with respect to the relative momentum vector of the two particles. Scattering off the long/short face would produce a scattering angle of $\pm \pi / 2$, respectively, with the long face yielding a much larger scattering amplitude. Thus, the fermionic amplitude at $\pi / 2, \tilde{F}(\pi / 2)=f(\pi / 2)-f(-\pi / 2)$, would be nonzero. Clearly, the standard wisdom that identical fermions cannot scatter at angle $\pi / 2$ applies only to parity-invariant potentials.

Although in potential scattering the ability to perform a partial wave decomposition implies $\mathbf{P}$ invariance, one is certainly free to contemplate a situation where $\delta_{m}$ is not an even function of $m$, in which case fermions can scatter at angle $\pi / 2$. As will be seen, this is exactly what occurs when a statistical interaction is added: one has rotational invariance, so that a partial wave decomposition is possible, yet $\mathbf{P}$ invariance is broken, so that in general $\delta_{m} \neq \delta_{-m}$.

Pure AB scattering and the scattering of free anyons are both described by the Hamiltonian

$$
H=-\frac{1}{2}\left(\frac{\partial^{2}}{\partial r^{2}}+\frac{1}{r} \frac{\partial}{\partial r}+\frac{1}{r^{2}}\left(\frac{\partial}{\partial \theta}-i \alpha\right)^{2}\right)
$$

Here, $\alpha$ represents the flux in $\mathrm{AB}$ scattering in units of the flux quantum, and the statistical parameter in anyon scattering (normalized so that $\alpha=1$ represents transmutation between fermions and bosons). Again, one looks for a solution in the form of an incident wave plus scattered wave. Technically, the incident wave must 
be a plane wave "modulated" by an additional phase due to the vector potential in order to describe a uniform particle current (specifically, $\psi_{\mathrm{inc}}=\exp i(k r \cos \theta+$ $\alpha \theta)$ ), but, as has been emphasized by Hagen [15], we can be cavalier about this since a "naive" incident plane wave describes the correct covariant particle current as $r \rightarrow \infty$, and, indeed, the scattering amplitude is unaffected by the choice of incident wave except in the form of a $\delta$-function in the forward direction. In fact, in what follows we will simplify life by ignoring contributions to $f(\theta)$ which represent a $\delta$-function in the forward direction.

The solution of this problem is well known $[16,15]$ : the phase shifts, scattering amplitude and differential cross-section for $\mathrm{AB}$ scattering are

$$
\begin{aligned}
\delta_{m}^{A B}(\alpha) & =\frac{\pi}{2}(|m|-|m-\alpha|), \\
f_{\alpha}^{A B}(\theta) & =-\frac{\sin \alpha \pi}{\sqrt{2 \pi i k}} \frac{e^{i(N+1 / 2) \theta}}{\sin \theta / 2} \\
\frac{d \sigma}{d \theta}^{A B} & =\frac{\sin ^{2} \alpha \pi}{2 \pi k \sin ^{2} \theta / 2} .
\end{aligned}
$$

where $N$ is the integer part of $\alpha$, while for boson-based anyon scattering,

$$
\begin{aligned}
F_{\alpha}^{A B}(\theta)=f_{\alpha}^{A B}(\theta)+f_{\alpha}^{A B}(\theta+\pi) & =-\frac{\sin \alpha \pi}{\sqrt{2 \pi i k}} \frac{2 e^{i(N+1 / 2) \theta} e^{i(-)^{N} \theta / 2}}{\sin \theta} \\
{\frac{d \Sigma_{\alpha}}{d \theta}}^{A B}=\left|F_{\alpha}^{A B}(\theta)\right|^{2} & =\frac{2 \sin ^{2} \alpha \pi}{\pi k \sin ^{2} \theta}
\end{aligned}
$$

It is interesting to observe that, in spite of the fact that the Hamiltonian is not $\mathbf{P}$ invariant, the scattering cross-section is.

One important property of $f_{\alpha}^{A B}(\theta)$ is its periodicity (up to a phase) under an integer change of $\alpha$. This is due to the fact that only the fractional part of the flux is physically relevant in $\mathrm{AB}$ scattering. Thus, the differential cross-section must 
be invariant under $\alpha \rightarrow \alpha+n$, although the scattering amplitude itself can (and does) change by a phase: one finds

$$
f_{\alpha+n}^{A B}(\theta)=(-)^{n} e^{i n \theta / 2} f_{\alpha}^{A B}(\theta) .
$$

This relation, expressed in terms of phase shifts, is

$$
\delta_{m}^{A B}(\alpha+n)=\delta_{m-n}^{A B}(\alpha)-\frac{n \pi}{2} .
$$

Now let us suppose a cylindrically symmetric potential $V(r)$ is added, and study scattering as $\alpha$ is varied, holding the potential fixed. Again we can perform a partial wave expansion, obtaining phase shifts $\Delta_{m}^{V}(\alpha)$ which, in principle, can be determined in terms of the potential. These phase shifts obey the same periodicity requirement as the $\delta_{m}^{A B}(\alpha)$ :

$$
\Delta_{m}^{V}(\alpha+n)=\Delta_{m-n}^{V}(\alpha)-\frac{n \pi}{2}
$$

It is useful to separate $\Delta_{m}^{V}$ into the $\mathrm{AB}$ phase shift and a residual one due to the potential:

$$
\Delta_{m}^{V}(\alpha)=\delta_{m}^{A B}(\alpha)+\delta_{m}^{V}(\alpha)
$$

The key observation is that $\delta_{m}^{V}$, although due to the potential must necessarily depend upon $\alpha$. This can be seen most easily by substracting the periodicity conditions (4) and (5); one obtains

$$
\delta_{m}^{V}(\alpha+n)=\delta_{m-n}^{V}(\alpha)
$$

Although convenient, this separation is not without its dangers. For instance, in Refs. 10,12, a "conventional" interaction between anyons was parameterized by 
an additional phase shift $\delta$ in the lowest partial wave $m=0$, and $\mathbf{P}$ violation in the resulting scattering as $\alpha$ varied was discussed. However, keeping the residual phase shifts $\delta_{m}^{V}$ fixed as $\alpha$ varies implies that the conventional interaction must evolve as $\alpha$ changes, in a rather complicated way. Indeed, if the added phase shifts are an even function of $m$, the added interaction is $\mathbf{P}$ invariant at $\alpha=0$, but away from this point the conventional interaction (suitably evolved so as to keep the added phase shifts constant) necessarily violates parity.

This can be clearly demonstrated in a simple way for integer values of $\alpha$, as follows. As stated above, for conventional scattering $(\alpha=0), \mathbf{P}$ invariance implies that $\delta_{m}$ is an even function of $m$; in the present context, thus, $\delta_{-m}^{V}(0)=\delta_{m}^{V}(0)$. The periodicity requirement $(6)$ of $\delta_{m}^{V}(\alpha)$ then implies the following "shifted evenness" requirement for any $\mathbf{P}$-conserving potential at nonzero integer values of $\alpha$ :

$$
\delta_{n+m}^{V}(n)=\delta_{n-m}^{V}(n)
$$

The hypothetical potential which produces $\alpha$-independent phase shifts will certainly not obey (7), since its phase shifts are an even function of $m$. (It takes but a moment to convince oneself that phase shifts which are even both about $m=0$ and about $m=n \neq 0$ are pathological beyond all reason.) Thus, the potential must evolve to a parity-violating one for nonzero integer $\alpha$. For noninteger $\alpha$, while we have not proven that the potential must evolve to one which violates parity if the residual phase shifts are independend of $\alpha$, continuity arguments make it exceedingly plausible.

To make these ideas concrete, we can examine the case discussed in $[10,12]$, where the potential was parameterized by a phase shift in the lowest partial wave. 
At $\alpha=0$, such a phase shift is the result of a rather peculiar $\delta$-function potential if the other phase shifts are truly zero. A complete discussion would involve delving into the subject of self-adjoint extensions [17], but for our purposes a less mathematical approach will suffice [18]. Consider scattering in the presence of a cylindrical potential well of radius $a$ and depth $\lambda / \pi a^{2}$, in the limit $a \rightarrow 0$, whence the potential tends towards $\lambda \delta^{2}(\mathbf{r})$. This "regularized" $\delta$-function enables us to adopt the physically reasonable boundary condition that the wave function is finite at the origin (mathematically, this is equivalent to making a particular choice of self-adjoint extension) [19]. Keeping the momentum $k$ fixed, it is easy to show that, as $a \rightarrow 0$, the phase shift in the lowest partial wave goes to zero as $1 / \log k a$. However, if we consider the coefficient $\lambda$ to be itself a function of $a$, which goes to zero as $a \rightarrow 0$, then the lowest partial wave is given by

$$
\tan \delta_{0} \simeq-\frac{\pi}{2\left(\log \frac{k a}{2}+\gamma\right)+\frac{4 \pi}{\lambda}}
$$

This can be made nonzero if $\lambda$ goes to zero just quickly enough to cancel the divergent logarithm; specifically, if

$$
\lambda \rightarrow \frac{4 \pi}{D-2 \gamma-\log \frac{k^{2} r^{2}}{4}}
$$

where $D$ is a constant, then

$$
\tan \delta_{0} \rightarrow-\frac{\pi}{D}
$$

All other partial waves will be zero, essentially because the centrifugal barrier prevents them from coming into contact with the potential. 
It is a straightforward matter now to check how the phase shifts evolve when we turn on the statistical interaction; not surprisingly, one finds that $\delta_{0}$ drops to zero immediately once $\alpha$ becomes nonzero: the delicate balance between the two divergent terms in the denominator of (8) is lost. Furthermore, all the phase shifts remain zero until $\alpha=1$, at which point the first partial wave is nonzero and all others are zero, in agreement with (6).

Since the initial potential does not maintain constant phase shifts for nonzero $\alpha$, some other potential is required. It would be interesting to find this potential, but we have not managed to do so. (It would certainly be very unusual, since, according to the general arguments given above, we expect it to violate parity.)

A second concrete example of the above ideas is the example of hard-disk scattering [14], which is discussed below, and with which we will examine the question of justification of the MFA for the anyon gas.

\section{Anyons with hard-disk repulsion}

Consider the case of the scattering of two anyons whose interaction is described by a hard-disk repulsion within a region of size $a$ [14]. Parity violation in the scattering cross-section will enable us to use the ideas presented in Ref. [10] to address the question of the validity of the MFA.

The scattering amplitude $f_{\alpha}(\theta)$ can be determined in a rather conventional fashion; as mentioned above, it is useful to separate it into a pure $\mathrm{AB}$ piece, $f_{\alpha}^{A B}(\theta)$, and a new piece which owes its existence due to the hard-disk interaction:

$$
f_{\alpha}(\theta)=f_{\alpha}^{A B}(\theta)+f_{\alpha}^{H D}(\theta)
$$


The latter term can be written in terms of phase shifts $\delta_{m}^{H D}(\alpha)$, which are computed by imposing the boundary condition $\psi(a)=0$; explicitly, one finds

$$
\tan \delta_{m}^{H D}=\tan \delta_{|m-\alpha|}=\frac{J_{|m-\alpha|}(k a)}{N_{|m-\alpha|}(k a)} .
$$

These phase shifts clearly depend on the statistical parameter $\alpha$, and, moreover, satisfy the periodicity requirement (6), in accord with the discussion of the previous section. In fact, at low energies the tangent of the phase shifts have the following limiting behaviour:

$$
\tan \delta_{m}^{H D} \rightarrow \underset{k a \rightarrow 0}{\longrightarrow}\left\{\begin{array}{ll}
(k a)^{2|m-\alpha|} & m-\alpha \neq 0 \\
\log k a & m-\alpha=0
\end{array} .\right.
$$

If $\alpha=0$, the particles are normal bosons and the dominant partial wave is $m=0$; the potential thus gives approximately the scattering behaviour discussed in $[12,10]$. As $\alpha$ evolves towards 1 , the $m=1$ partial wave starts to contribute significantly and is, indeed, the dominant one beyond $\alpha=1 / 2$. When $\alpha=1$ we have a shift by one unit of the phase shifts compared with $\alpha=0$, in agreement with the previous section.

The hard-disk part of the scattering amplitude is

$$
f_{\alpha}^{H D}(\theta)=\frac{1}{\sqrt{2 \pi i k}} \sum_{m=-\infty}^{\infty} e^{i m \theta} e^{i \pi(|m|-|m-\alpha|)}\left(e^{2 i \delta_{m}^{H D}(\alpha)}-1\right) .
$$

This problem is a natural situation in which we can apply the method presented in Ref. 10 in order to check the validity of the MFA. We start by considering bosonbased anyons. The cross-section for the scattering of two anyons is given by

$$
\frac{d \Sigma_{\alpha}}{d \theta}=\left|f_{\alpha}(\theta)+f_{\alpha}(\theta-\pi)\right|^{2}
$$

for which an even integer $\alpha$ describes bosons while an odd one describes fermions. 
Our goal is to compute a mean scattering angle, and from it to compute a mean radius of curvature for a typical anyon trajectory. In order to proceed with the argument, we now have to evaluate the quantity

$$
X \equiv\left|\int d \theta \theta \frac{d \Sigma_{\alpha}}{d \theta}\right|
$$

which, once normalized by the total cross-section $\Sigma$, gives the mean radius of curvature $\bar{\theta}=X / \Sigma$. The mean length between scatterings is given by $L=1 / s \Sigma$ where $s$ is the anyon density. These two ingredients enable us to evaluate, if the mean scattering angle is small, the mean radius of curvature of the quasi-circular orbit, which is given by

$$
\bar{R}=\frac{L}{\bar{\theta}}=\frac{1}{s X}
$$

The number of particles contained whithin this roughly-circular orbit will then be

$$
Q \sim \bar{R}^{2}=\frac{k^{2}}{s X^{\prime 2}}
$$

where we have defined a new quantity $X^{\prime}=k X$, which depends only on the parameters $\alpha$ and $k a$.

The momentum $k$ can be estimated in the following way. The mean radius $\bar{R}$, which classically represents a length scale for the particle's trajectory, can be used to define at the quantum level the size of the region where the wave function is localized. The uncertainity principle then gives us a minimum momentum of $k \sim 1 / \bar{R}=s X^{\prime} / k$. The number of particles $Q$ can then be expressed in the simple 
form

$$
Q \sim \frac{1}{X^{\prime}} .
$$

The MFA is declared valid if $Q \gg 1$, since that implies that the graniness of the distribution of particles is on a much smaller scale than the length scale of the particles' trajectories. This gives us the criterion of validity

$$
X^{\prime}<X_{c}^{\prime} \text {, }
$$

where $X_{c}^{\prime} \ll 1$, the appropriate value being dictated by physical considerations.

From the definition of the mean angle (12) and using the scattering amplitudes (2) and (11), we can compute the cross-section $d \Sigma_{\alpha} / d \theta$, and thus the quantity $X^{\prime}$. We find the following rather unwieldy expression:

$$
\begin{aligned}
X^{\prime}= & 8 \sum_{l \neq l^{\prime}}(-)^{\frac{l+l^{\prime}}{2}} \frac{1}{l-l^{\prime}}\left[\frac{J_{+} J_{+}^{\prime}\left(J_{+} N_{+}^{\prime}-J_{+}^{\prime} N_{+}\right)}{\left(N_{+}^{2}+J_{+}^{2}\right)\left(N_{+}^{\prime 2}+J_{+}^{\prime 2}\right)}-\frac{J_{-} J_{-}^{\prime}\left(J_{-} N_{-}^{\prime}-J_{-}^{\prime} N_{-}\right)}{\left(N_{-}^{2}+J_{-}^{2}\right)\left(N_{-}^{\prime 2}+J_{-}^{\prime 2}\right)}\right] \\
+ & 8 \sum_{l, l^{\prime} \neq(0,0)}(-)^{\frac{l+l^{\prime}}{2}} \frac{2}{l+l^{\prime}} \frac{J_{-} J_{+}^{\prime}}{\left(N_{-}^{2}+J_{-}^{2}\right)\left(N_{+}^{\prime 2}+J_{+}^{\prime 2}\right)} \\
& \times\left[\left(N_{-} N_{+}^{\prime}+J_{-} J_{+}^{\prime}\right) 2 s c+\left(J_{-} N_{+}^{\prime}-J_{+}^{\prime} N_{-}\right)\left(c^{2}-s^{2}\right)\right] \\
& +\frac{8}{\pi} \sum_{l}\left\{J_{-} \frac{N_{-} s^{2}+J_{-} s c}{N_{-}^{2}+J_{-}^{2}} I_{l-2}-J_{+} \frac{N_{+} s^{2}+J_{+} s c}{N_{+}^{2}+J_{+}^{2}} I_{l}\right\},
\end{aligned}
$$

where the sums are over $l, l^{\prime}=0,2,4, \ldots$, and where we have used the notation $J_{ \pm}=J_{l \pm \alpha}(k a), J_{ \pm}^{\prime}=J_{l^{\prime} \pm \alpha}(k a), s=\sin (\alpha \pi)$ and $c=\cos (\alpha \pi)$. Furthermore, $I_{l}$ is given by

$$
I_{l}=\pi \sum_{k=1}^{l / 2} \frac{(-1)^{k}}{k}+\pi \ln 2 .
$$

(15) is sufficiently ugly that we must turn to a numerical analysis in order to extract the behaviour of $X^{\prime}$ as we vary the parameters $\alpha$ and $k a$. The resulting graphs are 
then visually analysed to determine the regions in the parameters $\alpha$ and $k a$ which satify the criterion of validity of the MFA (14). Without loss of generality, we can restrict the range of $\alpha$ to $[0,1], \alpha=0$ and $\alpha=1$ describing respectively bosons and fermions.

Fig. 1 presents a three-dimensional plot of $X^{\prime}$ in terms of the parameters $\alpha$ and $k a$. Fig. 2 diplays a contour plot of the same function where the solid region indicates the values of $\alpha$ and $k a$ satisfying the criterion of validity (14) (where we have chosen $X_{c}^{\prime}=0.1$ ). Fig. 3 shows a plot of $X^{\prime}$ in terms of $\alpha$ for fixed $k a$ (which has been put at the value $k a=0.1$ ).

By inspection of the plots diplayed in Fig. 1 and Fig. 2, we are able to conclude that the criterion of validy is assured in three different limits in the parameters: 1) $\alpha$ near 0, i.e. anyons near the bosonic regime; 2) $\alpha$ near 1 , i.e. anyons near the fermionic regime; 3) $k a$ very small for $\alpha$ arbitrary. The last condition is not very surprising since as $k a$ goes to zero the particles become free anyons, whose scattering does not violate parity [12]. The trajectory of an anyon is then apparently straight (on average) and the argument based on parity violation described above loses is utility for studying the validity of the MFA.

The plot displayed in Fig. 3 allow us to go much further in our analysis of the MFA since it shows not only the regions in the parameter $\alpha$ where the criterion of validity (14) is respected, but also gives an indication of the dependence of $X^{\prime}$ on $\alpha$ near $\alpha=0,1$. As we can see, one can expect $X^{\prime}$ to depend linearly on the statistical parameter $\alpha$ near the bosonic regime and quadraticaly on the statistical parameter $\tilde{\alpha}=1-\alpha$ (measuring the departure from Fermi statistics) near the fermionic regime. 
This can be understood analytically as follows. We are interested primarily in values of $k a$ considerably less than 1 , since on the one hand the momentum satisfies $k \bar{R} \sim 1$, and on the other hand the mean radius of curvature $R$ should be greater than $a$ in order for the whole approach of considering isolated scatterings of anyons to be reasonable. In the bosonic regime, $\alpha$ near $0, X^{\prime}$ has the limiting form

$$
X^{\prime} \rightarrow \frac{2 \pi^{3} \log 2}{(\gamma+\log (k a / 2))^{2}+\pi^{2}} \alpha,
$$

which clearly shows a linear dependence on the statistical parameter $\alpha$. The MFA is, therefore, deemed justified for

$$
\alpha \ll 1 . \quad \text { near bosons }
$$

In the fermionic regime, $\tilde{\alpha}$ near 0 , the situation is more complicated. Writing $k a=\epsilon$, for $\tilde{\alpha} \ll 1$, we find

$$
X^{\prime} \rightarrow \pi^{3} \epsilon^{2} \tilde{\alpha}\left(c \epsilon^{2}+d \tilde{\alpha}\right), \quad c, d \sim 1
$$

Thus, for $\tilde{\alpha} \ll \epsilon^{2}$, the dependence of $X^{\prime}$ on $\tilde{\alpha}$ is linear, while for $\tilde{\alpha} \gtrsim \epsilon^{2}$, it is quadratic. However, we are concerned with the dependence when $X^{\prime}$ is near its critical value $X_{c}^{\prime}$. For $\epsilon$ considerably smaller than 1, the appropriate dependence on $\tilde{\alpha}$ can easily be seen from (18) to be quadratic, indicating that the MFA is justified if

$$
\tilde{\alpha}^{2} \ll 1 . \quad \text { near fermions }
$$

In both cases, we recover the results obtained previously $[6,7,8,9]$, but perhaps in a more trustworthy way since self-consistency was not a part of our argument. 
In summary, we have discussed general features of Aharonov-Bohm scattering with an additional conventional interaction, with a particular emphasis on the parity violation which may be found in the scattering cross-section. The danger of parameterizing the added interaction by phase shifts was pointed out; the essential point is that the added phase shifts must depend on the statistical parameter. In the latter part of the paper, we applied the "asymmetric-scattering" method discussed in [10] on anyons with a hard-disk repulsion in order to extract a criterion for the validity of the MFA. In agreement with previous works, we found that the statistical interaction must be weak both for anyons near bosons and near fermions, the quantitative criteria being given by (17) and (19).

We thank T. Gisiger for help with the numerical work, and F. Wilczek for useful discussions. This work was supported in part by the Natural Science and Engineering Research Council of Canada and the Fonds F.C.A.R. du Québec.

\section{REFERENCES}

1. J.M. Leinaas and J. Myrheim, Nuo. Cim. B37, 1 (1977).

2. F. Wilczek, Phys. Rev. Lett. 48, 1144 (1982).

3. F. Wilczek, Phys. Rev. Lett. 49, 957 (1982).

4. For references and review, see, e.g., F. Wilczek, Fractional statistics and anyon superconductivity (World Scientific, 1990); A.P. Balachandran, E. Ercolessi, G. Morandi, A.M. Srivastava, Hubbard Model and Anyon Superconductivity (World Scientific, 1990).

5. D.P. Arovas, et al., Nucl. Phys. B251 [FS13], 117 (1985). 
6. Y-H. Chen, F. Wilczek, E. Witten and B. Halperin, Int. Jour. Mod. Phys. B3, 1001 (1989).

7. C. Trugenberger, Phys. Rev. D 45, 3807 (1992).

8. A. Zee, "Semionics: a Theory of High Temperature Superconductivity," in High Temperature Superconductivity, eds: K. Bedell, D. Pines and J.R. Schrieffer (Addison-Wesley, 1990).

9. J. McCabe and R. MacKenzie, Mod. Phys. Lett. A8, 1909 (1993).

10. D. Caenepeel and R. MacKenzie, Mod. Phys. Lett. A8, 1909 (1993).

11. X.G. Wen and A.Zee, Phys. Rev. B 41, 240 (1990).

12. J. March-Russell and F. Wilczek, Phys. Rev. Lett. 61, 2066 (1988).

13. K. Kiers and N. Weiss, Phys. Rev. D 49, 2081 (1994).

14. A. Suzuki, M.K. Srivastava, R.K. Bhaduri and J. Law, Phys. Rev. B 44, 10731 (1991).

15. C.R. Hagen, Phys. Rev. D 41, 2015 (1990).

16. Y. Aharonov and D. Bohm, Phys. Rev. 115, 485 (1959).

17. M. Reed and B. Simon, Methods of Modern Mathematical Physics, vols. 1 and 2 (Academic Press, N.Y., 1972); P. de Sousa Gerbert, Phys. Rev. D 40, 1346 (1989); P. de Sousa Gerbert and R. Jackiw, Comm. Math. Phys. 124, 229 (1989).

18. K. Hwang, Quarks, Leptons and Gauge Fields (World Scientific, 1982); R. Jackiw, "Delta-function potential in two- and three-dimensional quantum mechanics", in M.A.Bég memorial volume, A.Ali and P.Hoodbhoy, eds. 
(World Scientic, Singapore, 1991); R. MacKenzie, P.K. Panigrahi, M.B.

Paranjape, S. Sakhi, to be published, Theoretical and Experimental Physics.

19. Hagen has recently emphasized the utility of using regularized versions of the AB scattering problem; see C.R. Hagen, Int. Jour. Mod. Phys. A6, 3119 (1991). See also J. Grundberg, et al., Mod. Phys. Lett. B5, 539 (1991); S. Artz, et al., Phys. Lett. B267, 389 (1991).

\section{FIGURE CAPTIONS}

FIG. 1. Three-dimensional plot of $X^{\prime}$ in terms of the parameters $\alpha$ and $k a$.

FIG. 2. Contour plot of $X^{\prime}$ in terms of the parameters $\alpha$ and $k a$. The solid region indicates the values of $\alpha$ and $k a$ satisfying the criterion of validity (14), with $X_{c}^{\prime}=0.1$.

FIG. 3. Plot of $X^{\prime}$ in term of $\alpha$ for fixed $k a$ (which has been put at the value $k a=0.1)$. 
This figure "fig1-1.png" is available in "png" format from: http://arxiv.org/ps/hep-th/9405027v1 
Fig.1

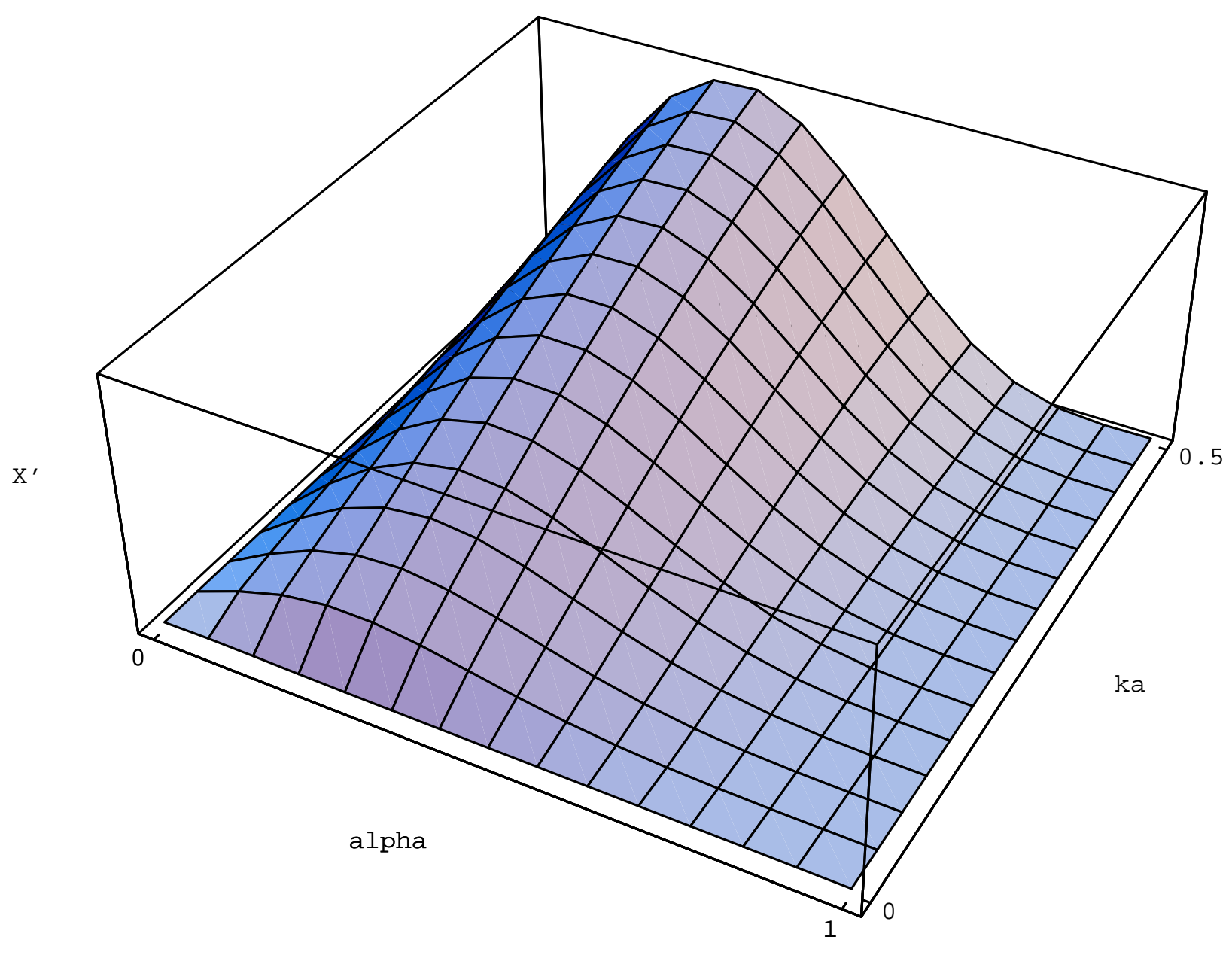


This figure "fig1-2.png" is available in "png" format from: http://arxiv.org/ps/hep-th/9405027v1 
Fig. 2

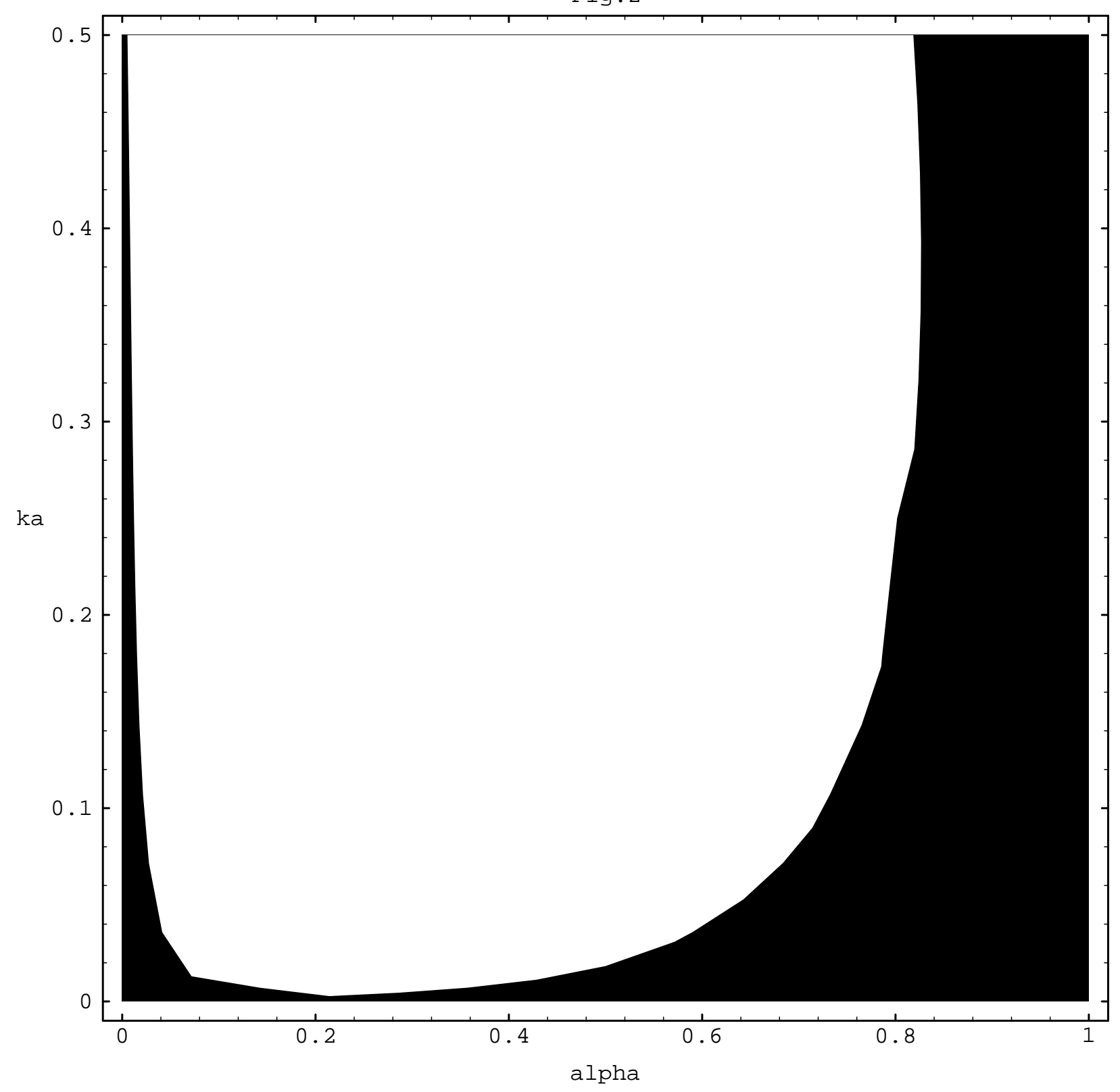


This figure "fig1-3.png" is available in "png" format from: http://arxiv.org/ps/hep-th/9405027v1 
Fig. 3

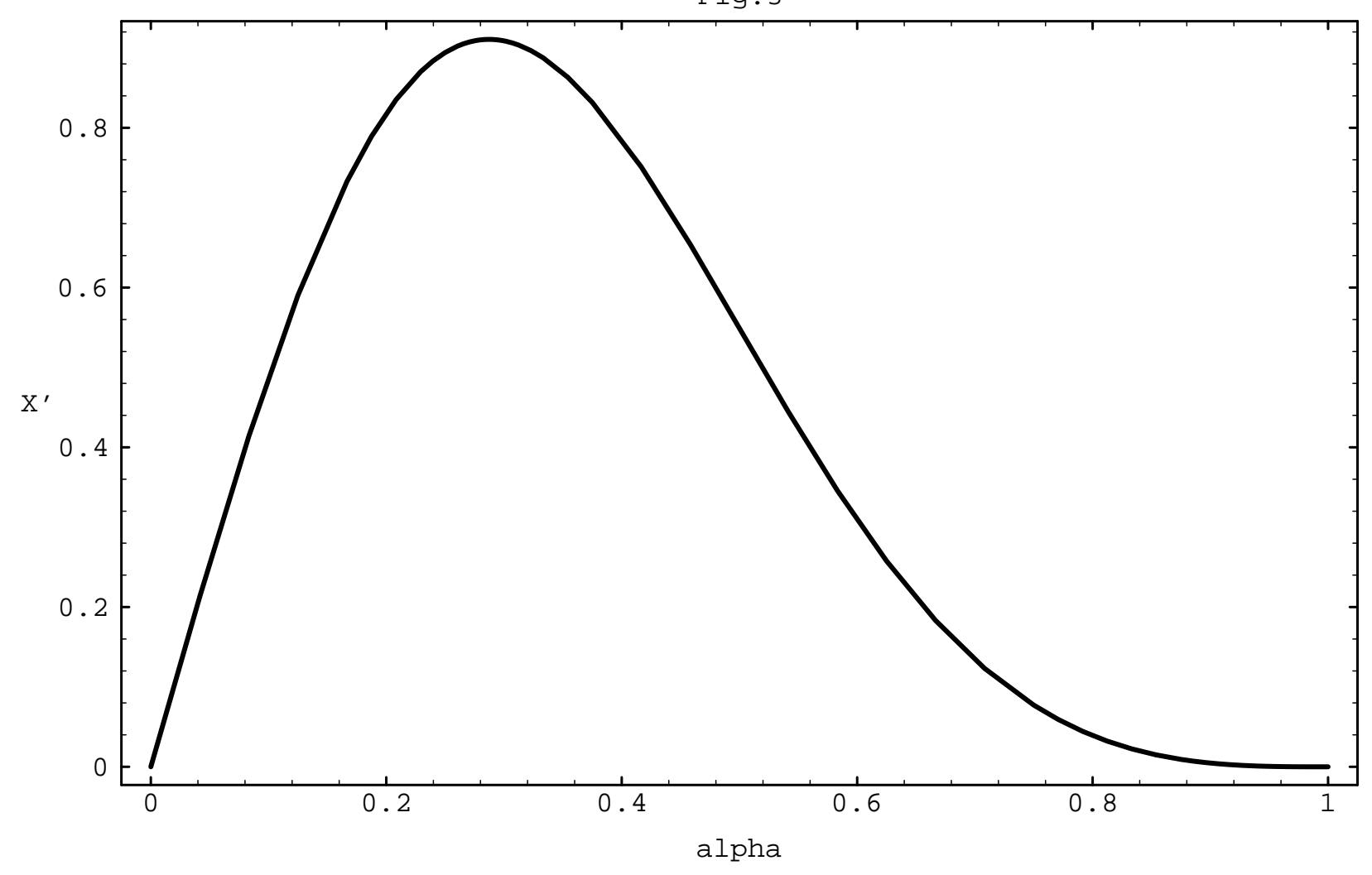

